\title{
Signal detection analysis of the effects of sequence duration on auditory matching to sample*
}

\author{
DONALD G. DOEHRING \\ uc.(iill Liniversity, Montreal, P.Q., Canada
}

Interstimulus interval (ISI) was varied from 250 to $2,500 \mathrm{msec}$ in an auditory matching-to-sample task in which a sample sound was followed by three choice sounds, one of which was the same intensity as the sample. Signal detection analysis of the results for eight Ss revealed that the pattern of discriminability $\left(d^{\prime}\right)$ among the three choice sounds was not significantly altered by variations of ISI. As the ISI became longer, however, false-alarm (FA) rate increased for the first choice and decreased for the last choice. It was concluded that for sequence durations assessed thus far, decreases in the $\mathrm{d}^{\prime}$ of choice sounds as a function of their remoteness from the sample sound are attributable to interference from intervening sounds rather than to passively decaying stimulus traces.

In previous studies of auditory matching to sample (Doehring, 1971: Doehring \& Libman, 1973), signal detection theory (Green \& Swets, 1966) was used to analyze serial position effects on three-choice sequence tasks, with hit $(\mathrm{H})$ rate calculated as the ratio of correct choices at a given serial position to all possible correct choices of that position, and false alarm (FA) rate as the ratio of incorrect choices of a given position to all possible errors at that position. Discriminability (d'), which was determined from the $\mathrm{H}$ and $\mathrm{FA}$ rates (Elliott, 1964), represented the relative difficulty of a choice at each position, independent of response bias (FA rate) for that position. This application of signal detection theory adds an important dimension to the study of temporal sequence effects in auditory discrimination, particularly since Ss tend to exhibit response biases (FA rate) which result in patterns of apparent accuracy $(H$ rate) that differ from patterns of discriminability (d') among the choice sounds. Ss tested in the previous studies were biased towards selecting the choice sound once-removed from the sample sound, regardless of the serial position of the sample. However, the choice sound immediately adjacent to the sample sound was most discriminable and the most remote sound was least discriminable (Doehring, 1973).

The present study was concerned with the effects on FA rate and $\mathrm{d}^{\prime}$ of varying interstimulus interval (ISI) in a matching-to-sample task where the sample preceded three choice sounds, one of which was the same intensity as the sample. The four $500-\mathrm{msec}$ tones were separated by ISIs of $250,500,1,500$, or $2,500 \mathrm{msec}$, resulting in total sequence durations of $2.75,3.5,6.5$,

*The writer wishes to thank Harriet Emerson, Ruth Libman, and Carolyn Cote for assistance in preparation, administration, and analysis of tests. This research was supported by Grant MA-1652 from the Medical Research Council of Canada. and $9.5 \mathrm{sec}$, as compared with a sequence duration of $7 \mathrm{sec}$ for $1-\mathrm{sec}$ test tones separated by $1-\mathrm{sec}$ intervals in the previous studies. If decrease in the $\mathrm{d}^{\prime}$ of choice sounds as a function of remoteness from the sample is largely attributable to interference from the intervening sounds, such variations of sequence length should have little effect. However, if $\mathrm{d}^{\prime}$ is decreased by a passive decay process, the effects of decay should be reduced for the shorter sequences and increased for the longer sequences. Since "our understanding of nonverbal memory is even poorer than that of verbal memory [Kintsch, 1970, p. 353]" and the few models of nonverbal auditory memory are restricted to two-sound sequences (cf. Kinchla \& Smyzer, 1967; Wickelgren, 1969), the results of this study should give some suggestion of the operation of relatively short-term auditory storage processes on a multiple-choice nonverbal recognition task in which Ss' decision processes are explicitly accounted for.

\section{METHOD}

Eight paid Ss, aged 17 to 24 , were tested. All had no more than $10 \mathrm{~dB}$ deviation from audiometric zero (ISO) in either ear. Test stimuli were tape-recorded sequences of four $2,000-\mathrm{Hz}$ tones $0.5 \mathrm{sec}$ in duration, with onset and decay times of $25 \mathrm{msec}$, presented at ISIs of $250,500,1,500$, and $2,500 \mathrm{msec}$. The choice sounds differed in intensity by 1.4 or $2.0 \mathrm{~dB}$, and were presented at a center intensity of $60 \mathrm{~dB}$ SPL. S pressed one of a horizontal series of pushbuttons to indicate his judgment of the serial position of the matching sounds and was given immediate knowledge of the correct position.

Each $S$ received 600 test trials. The trials were presented in eight series, one for each intensity difference at each ISI. Each series began with 12 practice trials, followed by 75 test trials. The matching sound was presented at each of the three different intensities and in each of the three serial positions on an equal number of test trials, with the positions and intensity values of the sounds otherwise randomized.

Ss were given four sessions, in each of which a series at $2.0-\mathrm{dB}$ intensity difference was presented, followed by a series at 1.4-dB intensity difference at the same ISI. The order of ISIs among sessions was counterbalanced among Ss. Testing was done in a sound-treated room. S listened through matched TDH 39 earphones.

\section{RESULTS}

Mean $\mathrm{H}$ rate, $\mathrm{FA}$ rate, and $\mathrm{d}^{\prime}$ for each position at each ISI are shown in Table 1 . There was no significant difference among ISIs in average accuracy of matching, which ranged from $\mathrm{H}$ rates of .45 for the $1,500-\mathrm{msec}$ interval to .49 for the $500-\mathrm{msec}$ interval. In accordance with previous results (Doehring, 1973), $\mathrm{d}^{\prime}$ for combined interstimulus intervals was largest for the choice sound closest to the sample (Position 1) and smallest at the most remote sound (Position 3). However. this systematic decrease of $\mathrm{d}^{\prime}$ occurred only for the longest and shortest ISIs, and neither the positional differences 
Table 1

Mean Hit (H) Rate, False Alarm (FA) Rate, and Discriminability $\left(d^{\prime}\right)$ for the Eight Ss at Each of the Four Interstimulus Intervals

\begin{tabular}{llccc}
\hline & & \multicolumn{3}{c}{$\begin{array}{c}\text { Serial Position } \\
\text { of Choice Sounds }\end{array}$} \\
\cline { 3 - 5 } & $\begin{array}{c}\text { Interstimulus } \\
\text { Interval (msec) }\end{array}$ & 1 & 2 & 3 \\
\hline \multirow{4}{*}{ H Rate } & 250 & .41 & .57 & .45 \\
& 500 & .44 & .55 & .47 \\
& 1500 & .42 & .50 & .44 \\
& 2500 & .48 & .56 & .36 \\
& Combined Intervals & .44 & .55 & .43 \\
& 250 & .18 & .35 & .26 \\
FA Rate & 500 & .21 & .31 & .24 \\
& 1500 & .26 & .30 & .26 \\
& 2500 & .24 & .34 & .22 \\
& Combined Intervals & .22 & .33 & .25 \\
& 250 & .70 & .57 & .52 \\
d & 500 & .66 & .63 & .63 \\
& 1500 & .47 & .53 & .50 \\
& 2500 & .68 & .60 & .41 \\
& Combined Intervals & .63 & .58 & .51 \\
\hline
\end{tabular}

for combined ISIs nor the interaction of positions and ISIs were significant. There was, however, a significant difference among positions in $F A$ rate $(F=10.12$, $\mathrm{p}<.01$ ), resulting from a significantly higher FA rate for Position 2 than for Positions 1 and $3(p<.01$, Newman-Keuls test). This is consistent with previous findings (Doehring, 1971, 1973). There was also a significant interaction of positions and ISIs for FA rate $(\mathrm{F}=3.10, \mathrm{p}<.05)$. As the ISI grew longer, FA rate increased for Position 1 and decreased for Position 3, i.e., Ss adjusted their decision criterion slightly in favor of early choices for long ISIs and late choices for short ISIs. A significant difference among positions $(\mathrm{F}=10.13, \mathrm{p}<.01)$ and a significant Position by ISI interaction $(\mathrm{F}=2.93, \mathrm{p}<.05)$ for $\mathrm{H}$ rate can, in the absence of significant position effects for $\mathrm{d}^{\prime}$, be said to directly reflect the Ss' significant response biases.

\section{DISCUSSION}

Both absolute and relative discriminability of choice sounds were surprisingly resistant to variation of sequence duration. Neither the shortening nor the lengthening of the sequence with respect to the 7-sec duration used in previous studies affected the pattern of $d^{\prime}$ among choice sounds in such a way that any inferences could be drawn about the differential decay of stimulus traces in nonverbal auditory memory. If anything, the pattern of decreased discriminability from the adjacent to the remote choice that occurred for both the shortest and longest sequence durations in the present study was the same as that found for the 7-sec duration of the previous studies. This suggests that the decrease in $\mathrm{d}^{\prime}$ as a function of remoteness of the choice sounds found previously (Doehring, 1973) was attributable to interference from intervening sounds rather than to passively decaying traces. A wider range of sequence durations is needed to assess this aspect of nonverbal auditory memory. There should be a duration brief enough so that sequences are processed as a single pattern with minimal decay and interference effects, as well as a sufficiently long duration so that either the decaying of traces severely impairs performance or some form of coding is required. The pattern of $d^{\prime}$ should alter appreciably at both such limits of performance.

The consistent elevation of FA rate at the position once removed from the sample has been noted in all groups of adults tested previously (Doehring, 1971, 1973; Doehring \& Libman, 1973). There is no ready explanation for this consistent response bias. The shifts in FA rates for the first and third choices as a function of ISI in the present study may indicate that Ss found that it required less effort to choose later sounds as matching the sample at short durations and to choose earlier sounds as matching the sample at long durations. Thus, the variation of sequence durations appears to have affected decision strategy but not discriminability. Further study is needed to obtain more information about how stimuli are processed as a function of serial order and time on auditory nonverbal recognition tasks, and how such processing relates to that of auditory verbal stimuli.

\section{REFERENCES}

Doehring, $D$, G Serial order effects in auditory discrimination. Perception \& Psychophysics, 1971, 10, 137-141.

Doehring, D. G. Signal detection analysis of serial order effects in auditory matching to sample. Unpublished manuscript, McGill University, 1973.

Doehring, D. G., \& Libman, R. A. Signal detection analysis of auditory discrimination by children. Unpublished manuscript, McGill University, 1973.

Elliott, P. B. Tables of $\mathrm{d}^{\prime}$. In J. A. Swets (Ed.), Signal detection and recognition by human observers. New York: Wiley, 1964. Green, D. M., \& Swets, J. A. Signal detection theory and psychophysics. New York: Wiley, 1966.

Kinchla, R. A., \& Smyzer, F. A diffusion model of perceptual memory. Perception \& Psychophysics, 1967, 2, 219-229.

Kintsch, W. Models for free recall and recognition. In D. A. Norman (Ed.), Models of human memory. New York: Academic Press, 1970.

Wickelgren, W. A. Associative strength theory of recognition memory for pitch. Journal of Mathematical Psychology, 1969, 6, 13-61.

(Received for publication May 1, 1973.) 